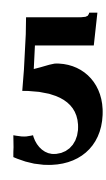

\title{
EDUCACIÓN Y JÓVENES EX-TUTELADOS: REVISIÓN DE LA LITERATURA CIENTÍFICA ESPAÑOLA
}

\author{
(EDUCATION AND YOUNG PEOPLE LEAVING CARE: SCIENTIFIC \\ LITERATURE REVIEW IN SPAIN)
}

Carme Montserrat Boada y Ferran Casas Aznar Universidad de Girona

\section{RESUMEN}

¿Cuál es el nivel educativo de los jóvenes que han pasado por el sistema de protección social a la infancia? En la mayoría de los países no se puede contestar esta pregunta. No existen datos sistemáticos ni estadísticos oficiales, exceptuando el Reino Unido. En el presente artículo se realiza una revisión de la literatura científica española a través de estudios y programas que aportan datos indirectos o directos sobre la escolarización y el nivel formativo de los jóvenes de población general en contraste con los del sistema de protección. Algunos de estos trabajos nos indican que estos jóvenes tienen un nivel educativo bajo y un abandono escolar prematuro muy acentuado comparado con la población general, siendo ambos factores frecuentemente citados como de riesgo a la exclusión social. La invisibilidad estadística en la que se encuentra el colectivo de niños y niñas tutelados y más concretamente la referida a su educación, viene acompañada de una escasez de investigación y de programas orientados a la inclusión de estos jóvenes en los distintos niveles de educación. Esta revisión persigue abrir un debate hoy por hoy poco implantado pero necesario.

\section{ABSTRACT}

What is the educational level of young people leaving care? In most countries we can not answer this question. There are no systematic or official statistical data, except in the case of the United Kingdom in the recent years. In 
this article we do a review of Spanish literature through studies and programs that provide direct or indirect data on the education and training level of young people in general population in contrast to children in care or young people leaving care. Some of these studies indicate that children in care have a low educational level and an early school leaving in compared with the general population, two factors often cited as risk to social exclusion. The statistical invisibility related to young people leaving care and specifically referring to his education, is accompanied by a shortage of research and programs focusing on the inclusion of young people in various levels of education. This review aims to open now a necessary debate.

\section{POLÍTICAS EDUCATIVAS Y DE BIENESTAR}

En la mayoría de los países europeos, los principios generales de educación contemplados en sus legislaciones hacen hincapié en la importancia de la igualdad de acceso y de oportunidades para todos los niños y niñas. Asimismo, la educación es un derecho contemplado en la Convención sobre los Derechos del Niño de Naciones Unidas (1989).

Desde distintos ámbitos de las políticas de la Unión Europea (UE) se plantean acciones de apoyo al acceso a la educación para los jóvenes de medios desfavorecidos, aunque ninguna se refiere de manera explícita a la población que proviene del sistema de protección infantil. La Estrategia de Lisboa (2000) adoptó el objetivo a alcanzar por los estados miembros de la UE en el 2010 de "convertirse en la economía basada en el conocimiento más competitiva y dinámica del mundo, capaz de crecer económicamente de manera sostenible con más y mejores empleos y con mayor cohesión social". Asimismo, el Consejo Europeo (de la UE) señaló que esos cambios exigían no solamente "una transformación radical de la economía europea", sino también «un programa ambicioso de modernización del bienestar social y de los sistemas educativos". La importancia de las dimensiones sociales de este compromiso fue reafirmada en la Conferencia de Berlín en 2003 y en el Comunicado de Bergen en 2005. Ahora bien, en el último informe referido a la evolución de los estados miembros de la UE hacia los objetivos de la "Estrategia de Lisboa en Educación y Formación 2010" (Commission of the European Communities, 2008), se observa que la situación de España respecto al abandono escolar prematuro no ha mejorado entre los años 2000 y 2007, presentando unos porcentajes del 29,1 y $31 \%$ respectivamente, unos de los más altos de la UE, especialmente teniendo en cuenta que en 2007 el porcentaje medio de los 27 países de la UE era del 14,8\% y el objetivo era el de llegar al 10\% en el 2010. En la reunión de jefes de estado y primeros ministros de la UE del pasado 14 de marzo de 2008, se le recordó a España la necesidad de que emprendiera medidas urgentes para redirigir la situación, dado que la tendencia de los últimos años no parecía que fuera a la baja. 
Una de las respuestas del gobierno español por acercarse a la consecución de estos objetivos fue, en 2006, la promulgación del Real Decreto $1631 / 2006$, por el que se establecen las enseñanzas mínimas correspondientes a la Educación Secundaria Obligatoria (ESO), y su puesta en marcha el presente curso 2008-09, permitiendo una cierta diversificación del currículo desde el tercer curso de la Educación secundaria obligatoria. Otra de las principales novedades que incorporaba la LOE son los Programas de Cualificación Profesional Inicial (PCPI), sustituyendo los Programas de Garantía Social, dirigidos a los alumnos de 16 años que no han obtenido el título en Educación Secundaria Obligatoria, para que tengan la oportunidad de obtener una acreditación profesional así como el título, mediante la realización de un módulo voluntario.

En 2005, como parte también de la estrategia de Lisboa para promover el crecimiento y el empleo, la UE adoptó un "Pacto Europeo para la Juventud", que reconoció que la integración de los jóvenes en la sociedad y la vida laboral "sólo podrán alcanzarse si los jóvenes adquieren las capacidades, las competencias y los conocimientos adecuados a través de una educación y una formación pertinentes y de gran calidad. Para ello es indispensable eliminar determinados obstáculos como las situaciones de pobreza y exclusión social que pueden afectar a los jóvenes. Es necesario asimismo suprimir las desigualdades entre los sexos, ya que las jóvenes están más expuestas al desempleo y a la pobreza, mientras que la tasa de abandono prematuro de la escuela es mayor entre los jóvenes".

Respecto a las políticas de bienestar y protección social, el Plan estratégico nacional de infancia y adolescencia 2006-2009 marcaba también el objetivo de prevenir el fracaso y promover el éxito escolar, dedicar los recursos necesarios para evitar el abandono escolar, y atender a la diversidad compensando las desigualdades, apoyando a los programas para los alumnos con necesidades educativas especiales y alumnos extranjeros. Entre los grupos más vulnerables no se encontraba mención alguna del colectivo de niños y niñas tutelados.

Tampoco en la última modificación legislativa de ámbito estatal, la Ley Orgánica 1/1996 de Protección Jurídica del Menor, se mencionaba nada concreto acerca de los procesos educativos de los niños y niñas bajo la tutela de la Administración. En cambio, cabe destacar los avances en política social en las tres últimas décadas en la línea de seguir implementando acciones bajo el paradigma de la normalización (Casas, 1998). En este sentido, han dejado de funcionar las escuelas ubicadas dentro de los centros residenciales, potenciando así la inclusión de los niños y niñas en los contextos escolares existentes en la red de servicios normalizados. 
Ahora bien, ¿Cual es la trayectoria educativa de los niños y niñas dentro del sistema de protección social a la infancia? Ni tan sólo podemos contestar la pregunta de qué tipo titulaciones obtienen o cuantos finalizan con éxito la educación secundaria obligatoria. Este tema de intersección entre departamentos (Educación y Bienestar), acaba siendo un conjunto vacío de datos, una tierra de nadie.

En este artículo se presenta una revisión de la literatura científica española ${ }^{1}$, con el objetivo de identificar los estudios realizados durante la década 1999-2009 que se hayan centrado total o parcialmente, o bien en la situación escolar de los niños y adolescentes bajo tutela administrativa, o bien en los jóvenes que han pasado por el sistema de protección a la infancia y su recorrido educativo y de formación. En el apartado siguiente se resumen los resultados más relevantes de los estudios identificados en función de estos parámetros. Bajo el tercer título se discuten, interpretan y comparan los resultados expuestos, aportando de forma colateral la referencia a algunos estudios más. Finalmente en el último apartado se redactan unas reflexiones finales para abrir un debate.

\section{ESTUDIOS ESPAÑOLES SOBRE EL NIVEL EDUCATIVO NIÑOS Y NIÑAS Y JÓVENES DE POBLACIÓN GENERAL EN CONTRASTE CON LA POBLACIÓN TUTELADA Y EX-TUTELADA.}

A efectos de la presente revisión los estudios identificados han sido agrupados en 3 bloques: (1) estudios referidos a los itinerarios educativos y formativos de los adolescentes y jóvenes españoles, (2) estudios referidos a la escolaridad de los niños y niñas tutelados por el sistema de protección social, y (3) estudios sobre los itinerarios educativos y formativos de los jóvenes extutelados.

\subsection{Estudios españoles referidos a los itinerarios formativos de los jóvenes}

Este bloque tiene por objetivo exponer los resultados de estudios recientes (2006-08) que aportan datos sobre los itinerarios educativos de niños y jóvenes de población general pero que a su vez incluyen resultados y conclusiones sobre la franja de población que fracasa o que abandona el sistema educativo de forma prematura. A este fin se han seleccionado las publicaciones de Navarrete (2007), Merino y García (2007) y Melendro (2007). Para aportar una referencia de contexto se hace mención también al Informe PISA 2006 (CSASE, 2007a). 
El trabajo dirigido por Navarrete (2007) se centra en los jóvenes y su paso por el sistema educativo, e incluye tanto datos generales de España, como desglosados por Comunidades Autónomas (CCAA). El nivel de formación de los jóvenes de 15 a 19 años (2001) aporta porcentajes del 32,9\% con estudios primarios, el 40,4\% con los estudios de ESO, el 18\% de Bachillerato, el 3,6\% de Ciclos Formativos de Grado Medio (CFGM) y en Ciclos Formativos de Grado Superior (CFGS) el 1,9\%. La media nacional de alumnos con título de graduado en secundaria es del 71,4\%. Se observan diferencias en el nivel de escolarización por sexos: el 78\% de las mujeres obtienen el título de Graduado en Secundaria después de cursar la ESO, y el $63,1 \%$ de los hombres. La tendencia es similar por lo que se refiere al título de Bachillerato (52,8\% de mujeres y $36,2 \%$ de hombres), un patrón que se mantiene en los estudios de nivel universitario.

Este estudio plantea varios grupos de factores explicativos del fracaso escolar que más adelante retomaremos con los niños y niñas tutelados: a) factores estructurales y de cambio social, b) factores del sistema educativo, y c) factores personales, que no psicológicos, de los propios alumnos. En él se defiende que no se debe seguir entendiendo el fracaso escolar como rendimiento insuficiente del alumnado, entre otras razones porque la concepción del rendimiento educativo en términos dicotómicos de éxito y fracaso no es suficiente para entender la realidad: "Mientras toda la comunidad educativa no entienda que si el sistema desprofesionaliza al educador/a, autoculpabiliza al individuo y deslegitima la escuela, se está reforzando la escalada social con base en los méritos escolares y se está generando un terreno adobado para el conformismo y la exclusión social" (Navarrete, 2007, 295).

El trabajo de Merino y García (2007) está centrado en Cataluña. Estos autores contextualizan y describen de forma clara la complejidad del tema de los itinerarios formativos. Parten de los datos que muestran como la participación de los adolescentes en la educación secundaria post-obligatoria es inferior a la de otros países de nuestro entorno, y del problema que esto supone, tanto por las repercusiones que tiene sobre el mercado laboral la oferta de jóvenes poco cualificados, como por las dificultades que tienen estos jóvenes para construir sus itinerarios formativos y, en consecuencia, para su inserción social y laboral. Una idea a resaltar es la distinción que hacen entre el término vía (las vías serian las posibilidades - limitadas - que ofrece un sistema educativo de cara a seguir un currículum diferenciado) y el término itinerario que viene a ser lo que los jóvenes construyen a lo largo de sus trayectorias vitales.

Desde la perspectiva de los autores, en el ámbito escolar los jóvenes escogen entre diferentes opciones a partir de la combinación de cuatro di- 
mensiones que luego nos serán útiles aplicadas a población tutelada: (i) La socialización diferencial, marcada por el origen familiar y por las expectativas asociadas a los grupos sociales, el género o el grupo etnicocultural; (ii) la orientación recibida desde el entorno más próximo, es decir la familia, el grupo de iguales o los tutores escolares; (iii) las variables escolares como son la experiencia escolar previa y la organización de la oferta educativa; y (iv) el análisis de coste, beneficio y riesgo.

Estos autores se encuentran con que no existe en nuestro país un dispositivo regular de recogida de información longitudinal que permita reconstruir los itinerarios que realizan los jóvenes a lo largo de los años. En su estudio transforman con metodología indirecta los datos oficiales referidos a matriculación y evaluación en datos de flujo. A partir de distintos diagramas de flujos, se puede consultar: (i) datos generales sobre los itinerarios educativos en Catalunya, (ii) los itinerarios educativos y formativos post-obligatorios de los jóvenes con el graduado en secundaria, (iii) los itinerarios formativos post-obligatorios de los jóvenes sin titulación, (iv) el acceso al mercado de trabajo y (v) los dispositivos específicos de inserción para los jóvenes sin calificación.

En cuanto a datos generales, se observa cómo en Cataluña (ver Diagrama 1) un $26 \%$ abandona el sistema educativo sin la obtención del graduado en secundaria. De éstos, incluso algunos no llegan a $4^{\text {a }}$ de ESO, y una tercera parte pasa por algún programa de garantía social (PGS), actualmente PCPI.

Un 37\% sigue la vía académica del bachillerato y se gradúa, de los cuales la mayoría llegan a la universidad, y el resto a ciclos formativos de grado superior. Un 14\% sigue la vía de los ciclos formativos de grado medio y obtiene el certificado, que sumados a los que han cursado algún un ciclo formativo de grado superior, llegan a un $25 \%$ el total de graduados en la rama de formación profesional.

El resto, un 23\%, sale del sistema educativo sólo con el graduado de secundaria; han intentado continuar con la enseñanza post-obligatoria sin éxito. Según los autores, si al fracaso escolar de la ESO le añadimos estos estudiantes que empiezan el bachillerato o un ciclo formativo pero no obtienen el título correspondiente, entonces estamos ante la cifra de casi la mitad de la promoción que sale al mercado laboral con una escasa calificación, cifras similares a las de las últimas promociones de la EGB con el sistema anterior a la LOGSE. 


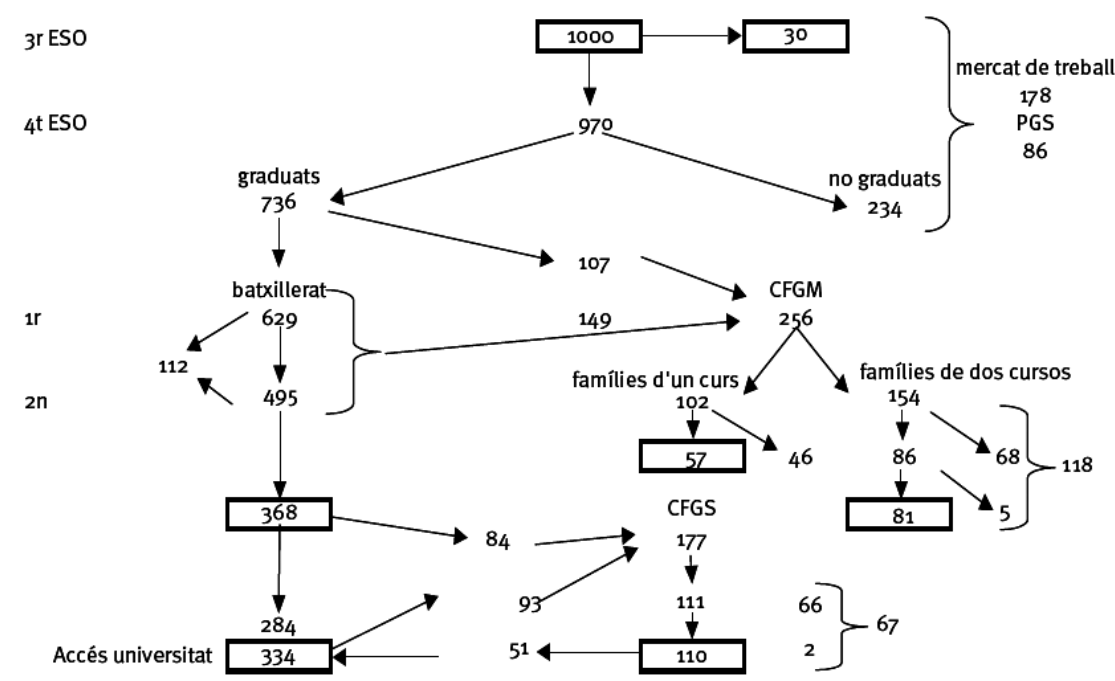

Fuente: Merino y García (2007).

Diagrama 1. Datos generales sobre los itinerarios formativos en Cataluña

El trabajo dirigido por Melendro (2007) incluye datos de ámbito estatal, pero en particular de la Comunidad Autónoma de Madrid. Este autor recoge información sobre principios y metodología de la intervención socioeducativa con adolescentes y jóvenes en situación de dificultad social. La publicación consta de dos partes: la primera expone los escenarios de los jóvenes en dificultad social, en los ámbitos educativo, laboral y social; la segunda expone un conjunto de estrategias de intervención socioeducativa a llevar a cabo con esta población y en diferentes ámbitos. En este trabajo se valora de forma positiva que en España haya un sistema educativo igualitario y poco excluyente, pero por el contrario se expone que los modelos escolares son poco flexibles. Señala también la tendencia a resaltar, por parte de diferentes agentes sociales, el protagonismo negativo al que a menudo se somete a los alumnos adolescentes. Aporta el dato a tener en cuenta por sus implicaciones prácticas sobre que la mayoría de los empresarios encuestados en el estudio de Madrid (Melendro, 2007) opinan que les son de gran utilidad los programas de acompañamiento de los jóvenes para su inserción laboral. Sobre los itinerarios para transitar a la vida adulta, se resalta la importancia del protagonismo de los jóvenes con el fin de que involucren en su propio cambio; es decir, se subraya que su participación es fundamental, cuestiones todas ellas de gran importancia si las revisamos desde los sistemas de protección a la infancia.

Para concluir este apartado, cabe destacar algunas valoraciones hechas en el último informe PISA (CSASE, 2007a), cuyos datos muestran que pese 
a que las puntuaciones medias del alumnado de 15 años se encuentran por debajo de la media de la OCDE, los alumnos españoles que pertenecen a familias con los índices sociales, económicos y culturales más bajos obtienen mejores resultados que los de sus homólogos de la OCDE. El informe aporta que la lectura de los datos debe tener en cuenta que hace 25 años más de la mitad de los jóvenes españoles de 15 años no estaban escolarizados y que en los últimos años se ha experimentado un crecimiento notable de la inmigración. Otro resultado a considerar es que la autonomía de los centros españoles es inferior a la del promedio de la OCDE, particularmente en los aspectos relacionados con la posibilidad de proponer sus necesidades de profesorado y de favorecer la promoción y la gratificación del mismo.

\subsection{Estudios españoles que hacen referencia a la educación de los niños y niñas tutelados por el sistema de protección social}

El criterio de selección en este apartado ha sido el identificar los estudios españoles que aportan resultados relevantes sobre aspectos referidos a la escolarización de los chicos y chicas tutelados. Para ello, se ha ampliado el intervalo de búsqueda a diez años con el fin de encontrar más trabajos. Estos son: Panchón (1999); Bravo y Del Valle (2003); Del Valle, López, Montserrat y Bravo (2007), Martín, Muñoz, Rodríguez y Pérez (2008) y ASJTET (2008).

En el trabajo dirigido por Panchón (1999), de los 354 cuestionarios sobre adolescentes entre 16 y 18 años aún en centro residencial, presentaban sólo un rendimiento escolar entre bastante y muy satisfactorio el 22,88\%. Un 45,2\% había pasado por episodios de absentismo escolar y sólo un 36,72\% había obtenido el graduado escolar, cifra muy inferior a la de la población general.

La relación que habían mantenido los adolescentes tanto con el profesorado como con sus pares era valorada entre bastante y muy satisfactoria para un $42,94 \%$. De la información obtenida en cuanto al nivel de escolari$\mathrm{dad} /$ formación en el momento del ingreso, un 69\% tenía un nivel que no correspondía con su edad. Así mismo se constataba que el nivel de motivación que presentaban era bajo en un 54\% de los casos, debido también a los largos períodos de absentismo escolar que habían tenido.

En 1999 el retorno a la familia de origen constituía para el 40\% la previsión de salida del centro. Un 18\% preveía ir a un piso compartido; un $7 \%$ a otro centro o residencia y había un 10\% sobre el que se estaba estudiando su situación. Ante la pregunta de si pensaban continuar los estudios, contesta- 
ban afirmativamente un 38,7\%; que "no" un 30,2\%, y "no lo sabían" un $31,1 \%$. Ante la pregunta de qué tipo de estudios se proponían continuar, un $12 \%$ quería obtener el graduado, un 5,1\% bachillerato, un 30\% formación profesional, y un $41 \%$ formación ocupacional. De la muestra seleccionada, 261 jóvenes accedieron a participar directamente en el estudio. De sus respuestas sobre los planes que tenían cuando salieran del centro, un 55\% tenían previsto trabajar, puesto que el trabajo les era imprescindible para independizarse.

En la publicación de Bravo y Del Valle (2003) donde se valoraba las redes de apoyo social de un grupo de adolescentes en atención residencial, se observaba que los jóvenes de las residencias confiaban más en los amigos del centro residencial y en amigos adultos (educadores, monitores) que en los procedentes del contexto escolar o de asociaciones, indicando la importancia de las relaciones mantenidas dentro del contexto residencial en detrimento de las relaciones con miembros de la comunidad; cuestión que los mismos autores apuntaban que debía replantearse en los programas de acogimiento residencial.

Sobre el rendimiento académico de los niños y niñas en acogimiento en familia ajena, Del Valle et al. (2007) apuntaban que los técnicos apreciaban que casi un tercio de los casos (31\%) presentaba muchos problemas, una cuarta parte tenía algunos problemas, un $29 \%$ eran buenos estudiantes y el 13\% restante mostraba necesidades especiales en el contexto escolar. En resumen, los datos indicaban que en torno al 57\% de niños y niñas en edad escolar mostraba bastantes dificultades en el contexto académico. Además, una quinta parte de los casos presentaba problemas de conducta en la escuela.

Los acogedores por su parte valoraban también que un 30\% de los niños acogidos presentaban muchos problemas, un $27 \%$ algunos problemas y un $13,3 \%$ presentaba necesidades educativas especiales. Tenían un buen rendimiento escolar el 30\%. Un 20\% presentaba a la vez problemas de comportamiento en la escuela. Los acogedores reclamaban apoyos urgentes en este ámbito.

En el trabajo de Martín et al. (2008) se analizaba la integración con los iguales en el contexto escolar de los menores en acogimiento residencial, tanto desde la perspectiva de los iguales como la de los profesores. Mediante la aplicación de un cuestionario sociométrico en 50 aulas y un protocolo de valoración para el profesorado, se comparó una muestra de 60 niños en acogimiento residencial con una muestra normativa de 843 sujetos. Los resultados mostraron que los niños y niñas en acogimiento residencial son significativamente menos elegidos y más rechazados por parte de los compa- 
ñeros a la hora de desarrollar tareas académicas. Por el contrario no se observaban diferencias significativas cuando se analizaban los rechazos y elecciones recibidas en el ámbito de las tareas de ocio.

También se indicaba que los niños y niñas en atención residencial eran percibidos por sus compañeros con atributos negativos para el desarrollo de actividades referidas a las tareas de instrucción que se desarrollan en el aula, como el tener malas relaciones con el profesorado, el ser agresivo o el querer llamar siempre la atención. Concluían los autores que estas conductas generaban inadaptación escolar. La valoración del profesorado coincidía con la que hacían los compañeros cuando valoraban lo que sucedía en clase, en cambio eran una fuente menos fiable cuando apreciaban lo que sucedía en las actividades de tipo informal que se desarrollaban fuera del aula.

Para finalizar este apartado destacamos los datos que nos aporta un estudio publicado por el Área de Apoyo a la Juventud Tutelada y Ex-tutelada (ASJTET, 2008) de la Generalitat de Catalunya sobre los chicos y chicas que se encuentran actualmente bajo medida legal y que tienen entre 15 y 18 años. En la recogida de datos participaron 77 de los 91 servicios, mayoritariamente centros residenciales de acción educativa, abarcando 478 jóvenes de un total de 512.

De las características de la muestra destaca que el $60 \%$ eran hombres, el $41 \%$ extranjeros, el 43\% tenía 17 años; el 76\% no padecía ningún tipo de patología (siendo el porcentaje más elevado el de los extranjeros) y el $80 \%$ de la población no tenía ningún tipo de relación con la justicia.

Sobre su nivel formativo, el $52 \%$ de la población no tiene el graduado de la escolaridad obligatoria (en esta situación se encontraban más chicos que chicas). El 5\% de la población había iniciado estudios previos a los universitarios o superiores (Bachillerato o Ciclo Formativo de Grado Medio), siendo también mayoritarias las mujeres y por nacionalidades, la española.

Por lo que se refiere a la prospección de salida del recurso de protección, destacaba que el 35\% de la población tenía como propuesta el ser atendido por el ASJTET y otro $29 \%$ continuaba en algún tipo de recurso de la Administración diferente del ASJTET. Se preveía que el 12\% se independizara y el $22 \%$ retornara con la familia.

Dado que el estudio muestra una cierta emergencia de orientaciones hacia estudios superiores (el 5\% de la población ha iniciado estudios secundarios post obligatorios), los autores apuntan que este hecho debería incorporarse en los programas de apoyo y de vivienda. A su vez reafirmaban la 
importancia y el crecimiento de la ASJTET en cuanto a número de jóvenes atendidos hasta los 21 años.

\subsection{Estudios españoles sobre los itinerarios formativos de los jóvenes ex-tutelados}

En este apartado el criterio de selección ha sido el de identificar trabajos referidos a población mayor de edad que ha salido del sistema de protección infantil y su situación respecto a su itinerario formativo, aunque sobre todo incluyen aspectos referidos al empleo, vivienda, delincuencia, drogadicción y parentalidad Se ha podido acotar la búsqueda en el período 2003-2008. Destacamos los estudios de Del Valle, Álvarez-Baz y Bravo (2003), Inglés (2005), Turró y Cortes (2005), Rodríguez Gómez (2006), FPA (2007) y García Barriocanal, Imaña y de la Herrán (2007), Las cuatro últimas incluyen datos de los jóvenes que se acogen a los programas de apoyo a los ex-tutelados.

Podríamos decir que la población que se acoge a los programas de mayores de 18 años presenta un sesgo en respeto a la población general de extutelados, especialmente en lo referente a la carencia de red de apoyo familiar y social que presentan y a la sobrerepresentación de chicos procedentes de centros residenciales. Por otro lado estos jóvenes deben aceptar unas normas; trabajar y/o estudiar, siempre con la idea de a corto plazo (máximo hasta los 21 años) procurarse una solvencia económica para poder ser autónomos.

El estudio de Del Valle et. al., (2003) incluye datos de jóvenes que estuvieron en centros de atención residencial, en la década de los 90', por lo que se aprecian diferencias con la población actual en centros, sobre todo con respecto a los años de estancia en los centros (que actualmente es menor) y a la tipología de la población atendida (con menos población autóctona en la actualidad). Presentan los resultados de una investigación realizada en España con una muestra de 260 jóvenes que habían estado en atención residencial cuando eran menores de edad. El seguimiento se llevó a cabo entre 1 y 9 años después de que hubieran dejado el centro residencial, y se evaluó su nivel de integración laboral y social, así como la incidencia de problemas relacionados con la marginación y la exclusión social.

De la población entrevistada, sólo un $5,8 \%$ continuaba estudiando y un $1,9 \%$ trabajaban y estudiaban al mismo tiempo. Se observó que el $60 \%$ de los jóvenes volvían al hogar familiar al salir de la residencia, donde se habían producido escasos cambios respecto a la situación que había desencadenado su ingreso años antes. Después de salir de las instituciones los jóvenes de- 
pendían mayoritariamente de su familia o de los servicios de bienestar social, pero a medida que se iban haciendo mayores abandonaban ese apoyo para ser más independientes. La variable más fuertemente asociada con posteriores problemas sociales fue el número de cambios de institución de los niños. Los problemas de desviación y marginación social afectaban a al $15 \%$ y lo que sí parecía más preocupante era que una cuarta parte dependía aún de los apoyos de los servicios sociales.

Tanto este estudio como el de García Barriocanal et al. (2007) constituyen los primeros estudios publicados sobre las consecuencias a largo plazo del impacto de la atención residencial en España, examinando tanto los indicadores positivos de la integración (la autonomía, el empleo, la vivienda, las relaciones sociales) y como los negativos (delincuencia, drogadicción, prostitución, prisión).

El trabajo dirigido por Inglés (2005) evaluó el conjunto de acciones y resultados de las actuaciones de 2 proyectos europeos: Mentor 15 y Ulises, destinados al acompañamiento hacia la autonomía e inserción sociolaboral de adolescentes y jóvenes tutelados por las administraciones de varias CCAA, incluido un manual de buenas prácticas. Referido a los estudios y la formación de los jóvenes del programa, se les preguntaba si les gustaría seguir estudiando: un 36\% respondió que sí, un 56\% que no, y un $8 \%$ no se pronunciaron sobre el tema. Los autores matizan que los que contestaron que Sí, respondía más a un deseo que a una situación real puesto que el trabajo era prioritario para su manutención y, en todo caso, eran más las chicas que los chicos. Con respecto a su nivel de formación, de los sujetos del programa el 18,3\% seguían algún curso; el resto, el 81,7\%, no. De los que estudiaban, una cuarta parte también trabajaba. Los que estudiaban vivían mayoritariamente en un piso asistido o con la familia extensa. De ellos, un 21,4\% estaba en la ESO, un 17,9\% en Bachillerato, un 19,6\% en un Ciclo Formativo de Grado Medio y un $16,1 \%$ en un programa de garantía social. Un 7,2\% está cursando estudios superiores (universitarios/no universitarios). Los que estaban en estudios universitarios tenían más de 22 años. Un 20,9\% seguían algún tipo de formación no reglada (peluquería, carpintería, mecánica, etc.), es decir formación breve y con un alto nivel de aplicabilidad. Este estudio incluyó entrevistas a informantes claves y a chicos y chicas, aportando datos en su mayoría descriptivos.

Turró y Cortes (2005) explican el desarrollo de un programa de intervención psicosocial con jóvenes ex-tutelados llevado a término en Catalunya y publicado en Chile. El objetivo general de la intervención planteada era crear un puente entre los jóvenes ex-tutelados por la Administración y la sociedad, mediante la figura de un referente, que facilitara su plena integración social. En Cataluña funciona desde hace años este programa de acompaña- 
miento a través de la Asociación Punto de Referencia (2008), que ha publicado también sus resultados.

Rodríguez Gómez (2006) hace una descripción del programa para mayores de 18 años ex-tutelados de la Comunidad Autónoma de Andalucía, en funcionamiento desde 1997; y aporta datos sobre: (i) el perfil general del grupo de jóvenes que participan en los recursos del Programa, (ii) el programa en sí con recursos a los que denominan de alta intensidad (incluye el recurso de vivienda) y de media y baja intensidad, y (iii) los resultados del programa. Aunque no aportan datos sobre los itinerarios formativos de estos jóvenes, de esta publicación resaltan como buenas prácticas unos macro encuentros que se hicieron de jóvenes que habían pasado por el programa y su participación activa en los mismos.

La Federación de Entidades con Proyectos y Pisos Asistidos FEPA (2007) presentó un informe-memoria de actividades realizadas por sus entidades federadas en el 2006. Esta federación se constituyó el diciembre de 1999 con el objetivo de aglutinar aquellas entidades que desarrollan proyectos para jóvenes tutelados y ex-tutelados a partir de los 16 años. En los datos consultados con respecto al grado de formación de los 1014 jóvenes atendidos en el 2006, se observa que un 3\% presentaba analfabetismo, un 37\% tenía un nivel básico de lectoescritura, un $18 \%$ cursó la ESO y no la acabó, un $28 \%$ obtuvo el graduado en ESO, un 11\% cursaba formación ocupacional, un 1,5\% ciclos formativos de grado medio y un 1,5\% bachillerato.

Dentro de su programa de formación para los jóvenes ex-tutelados durante el 2006, el 61\% de los cursos iniciados estaban orientados a la formación de un oficio, el 14\% orientado a la adquisición de los idiomas oficiales; el $23 \%$ a los ciclos formativos de grado medio y el $1 \%$ a grado superior.

Finalmente en el estudio de García Barriocanal et. al. (2007) se valoraba el grado de integración social de una muestra de 350 personas ex-residentes de centros de Madrid contemplando distintas variables como su situación laboral, de vivienda, núcleo de convivencia, etc., y contrastando algunos de los resultados con datos de población general presentados por el INJUVE. Incluía tanto resultados descriptivos como también la opinión de los ex-residentes. Por lo que ser refiere al nivel educativo, un $38 \%$ obtuvo el graduado escolar mientras estaba en el centro residencial y un $8 \%$ inició el bachillerato. Una vez fuera del centro, los que siguieron estudios fueron mayoritariamente de tipo profesional. Algunas de las opiniones de los jóvenes van en la dirección de aconsejar a los chicos y chicas que actualmente estén en centros "que aprovechen la oportunidad de estudiar porque después no van a tener los mismos recursos". 


\section{DISCUSIÓN DE LOS RESULTADOS EXPUESTOS POR LOS ESTUDIOS REFERIDOS}

El porcentaje de la población general que obtienen el graduado en ESO parece situarse alrededor del 72\% en España (Navarrete, 2007; Merino y García, 2007). Estos datos coinciden también con los del informe del CSASE (2007b) sobre la evaluación del alumnado matriculado en $4^{\circ}$ de ESO durante el curso 2003-2004 en Cataluña, dónde se graduaron el 69,6\% de los alumnos. Otro dato relevante es la diferencia por sexos en el sentido de que la no graduación afecta con más intensidad a los chicos. En dicho informe consta que los chicos que se graduaron representaban el 68\% y en cambio las chicas eran el 77\%, coincidiendo de nuevo con los datos aportados por Navarrete (2007) y con la preocupación plasmada también en el Pacto Europeo para la Juventud (2005).

Esta cuestión de género hace que se deba continuar enfatizando la mayor necesidad de apoyo a la formación académica entre los chicos (ASJTET, 2008).

Resalta, pues, que el número de estudiantes que no obtiene el certificado de educación obligatoria se encuentra aun muy por encima de la media de la UE. Ya hemos visto que el abandono escolar prematuro se situaba en España en el 2007 en el 31\% (European Commission, 2007).

Estos dos resultados, demasiados chicos y chicas sin el certificado de la ESO y el abandono escolar más acusado en los chicos, son importantes también a efecto de que constituyen el contexto en el que se desenvuelven los niños y niñas tutelados en España.

A partir de ahí, si intentamos comparar algunos de estos datos de población general con los de la población tutelada, las cifras son aún más alarmantes, como se puede observar en la Tabla 1:

\begin{tabular}{|l|c|c|}
\hline \multicolumn{1}{|c|}{ Graduados en: } & $\begin{array}{c}\text { Población } \\
\text { general (1) }\end{array}$ & $\begin{array}{c}\text { Población tutelada en aten- } \\
\text { ción residencial (15-18 años) }\end{array}$ \\
\hline ESO & $73,6 \%$ & $36,7 \%(2)$ \\
\hline CFGM/CFGS & $25 \%$ & $5 \%(3)$ \\
\hline Bachillerato & $37 \%$ & $\mathrm{~s} / \mathrm{d}$ \\
\hline Llegan a la Universidad & $28,4 \%$ & \\
\hline
\end{tabular}

Fuente: Elaboración propia a partir de varias fuentes (2008): (1) Merino y García, 2007; (2) Panchón, 1999; (3) ASJTET, 2008.

Tabla 1. Estimación de los chicos y chicas graduados entre la población general y la población tutelada, en Cataluña. 
El resultado es que observando estos primeros datos, comprobamos cómo la población tutelada se presenta como uno de los grupos más expuestos al abandono escolar prematuro sin ser identificado como tal por el propio sistema educativo. A su vez, se convierte en un grupo de riesgo a la exclusión social. Lo cual sugiere que esta población merecería una atención preferente o específica como la que pueden tener otros grupos: niños y niñas con discapacidades, los hijos de los inmigrantes recién llegados, los pertenecientes a grupos étnicos minoritarios o los niños y niñas con largas hospitalizaciones. La invisibilidad estadística, muchas veces argumentada bajo supuestos no discriminatorios (por ejemplo, el de evitar su etiquetaje social), ha prolongado en este caso una situación grave de falta de igualdad de oportunidades. En este sentido Lamarca y Barceló (2006) en su trabajo sobre menores en situaciones de especial vulnerabilidad, plantean que "en muchos campos existe una resistencia a ofrecer respuestas diferenciales; no respuestas iguales, sino compensatorias; que busquen la equidad, no la igualdad".

Entre la población tutelada graduada se observa una diferencia por sexos similar a la de la población general, habiendo más graduadas que graduados (ASJTET, 2008). No hay datos sobre porcentajes exactos de los que llegan a la universidad porque los pocos que lo consiguen, llegan con más de 22 años (Inglés, 2005), encontrándose ya fuera de los programas de apoyo, que son los que proporcionan escasas cifras de las que se disponen. Existen algunos estudios que ofrecen datos que complementan los hasta aquí expuestos. Por ejemplo, las dificultades escolares que presentan los niños y niñas en acogimiento familiar (Del Valle et al., 2007, Palacios 2007) y los que ingresan en un centro residencial. Entre los adolescentes que estaban en centros residenciales en España, un 69\% tenía un nivel que no correspondía con su edad (Panchón, 1999). También en estos estudios se muestran los problemas de motivación y de conducta que los niños presentaban en el entorno escolar. Martín et al. (2008) muestran el rechazo que sienten los niños en atención residencial por parte de sus compañeros de clase cuando se trata de desarrollar tareas académicas, factor que puede generar también inadaptación escolar. Bravo y Del Valle (2003) muestran la importancia de las relaciones mantenidas dentro del contexto residencial en detrimento de las relaciones con miembros de la escuela y barrio; factor que dificulta su integración social. Montserrat y Casas (2006) muestran que la satisfacción con la escuela por parte de los niños y niñas en acogimiento en familia extensa es el ámbito de la vida que puntúa más bajo. Bravo y Del Valle (2001) en su trabajo sobre evaluación de la integración social de jóvenes que habían vivido en centros residenciales de protección, hacen hincapié en la necesidad de contemplar, en los programas dirigidos a esta población, el objetivo de mantener a los chicos y chicas en su comunidad de origen con el fin de evitar la pérdida de amigos y los cambios innecesarios de escuela. 
Este compendio de dificultades atribuidas a los escolares tutelados por el sistema de protección da más pistas sobre lo que no funciona y de la necesidad de programas y estudios que apunten a la mejora en todos los aspectos - rendimiento, motivación, relaciones interpersonales, adaptación,...de su educación. Quizá se debería ahondar en el concepto expuesto por de Uriarte (2006) sobre la necesidad de adoptar la perspectiva de la resiliencia en la escuela con el objetivo de contribuir a superar las desigualdades y compensar los riesgos de exclusión social.

Otro tipo de resultados son los referentes a su transición a la vida adulta. Los datos de la Secretaria de Joventut (2008), nos muestran que en Catalunya la proporción de jóvenes emancipados en la franja de edad de 2024 años es sólo del 15,8\% (los no emancipados son el $84,2 \%$ ) y que en la franja de 25-29 años, un 54,6\% aún no lo está. En España los jóvenes se independizan de sus familias más tarde que en otros países europeos, de modo que no es hasta de 29 años que podemos decir que menos del 50\% vive con sus padres.

Estos datos contrastan con la población tutelada, que presenta otras cifras. En Panchón (1999), al cumplir los 18 años y salir del centro residencial en Catalunya se preveía que el $40 \%$ volvería con la familia de origen, y los demás debían afrontar procesos más rápidos de independencia. Por estas mismas fechas en España según el estudio Del Valle et al. (2003), el 60\% de los jóvenes volvían al hogar familiar al salir de la residencia, y a medida que se iban haciendo mayores abandonaban ese apoyo para ser más independientes, quedando una cuarta parte que dependía aún de los apoyos de los servicios sociales. Casi una década más tarde en el estudio del ASJTET (2008), se preveía un retorno a la familia sólo del $22 \%$, por lo tanto el programa de apoyo y transición a la independencia de estos jóvenes debía y debe atender a un número muy importante - y creciente - de jóvenes que deben afrontar la emancipación cuando la mayoría de sus pares en población general no lo hacen.

Es decir los jóvenes que provienen del sistema de protección deben independizarse rápidamente (a los 18 años) y sin demasiados apoyos sociales y familiares. La mayoría de ellos y de los profesionales que están en contacto con ellos se preguntan ¿Cómo pueden seguir estudiando en estas condiciones? Este dato explica la necesidad que tienen estos jóvenes de trabajar

Siguiendo en esta línea, resulta interesante aplicar a los jóvenes ex-tutelados la distinción entre vía e itinerario que hacen Merino y García (2007). Las vías que ofrece el actual sistema educativo, desde la perspectiva de la población tutelada resultan especialmente rígidas cuando se intenta permanecer "dentro" del sistema y no entrar en "vía muerta". De esta forma, los itinerarios 
que los jóvenes tutelados construyen a lo largo de sus trayectorias vitales para la obtención de algún título son a menudo muy complicados. También Melendro (2007) piensa que los modelos escolares son poco flexibles y por tanto constituyen un factor que contribuye a explicar el fracaso escolar.

Según los autores, estos itinerarios son fruto de la combinación de cuatro dimensiones que, aplicadas a la población tutelada, pueden ser: (i) su entorno familiar - lleno de dificultades - y las bajas las expectativas asociadas a su condición de tutelado; (ii) la orientación recibida desde el entorno más próximo, que acostumbra a ser la de seguir una vía formativa-laboral (iii) su experiencia escolar previa (a menudo con absentismo, falta de apoyo y de colaboración familiar) y la organización - rígida - de la oferta educativa; y (iv) el análisis de coste, beneficio y riesgo, que acaba decantándose en su caso de manera frecuente por la vía laboral en exclusiva.

Esta tendencia tan forzada hacia la vía laboral la vemos en la mayoría de los estudios. En el estudio de Panchón (1999), ante la pregunta de si pensaban continuar los estudios al salir de la residencia, contestaban afirmativamente un $38,7 \%$, pero sabiendo que un $30 \%$ se refería a formación profesional y un $41 \%$ a formación ocupacional. El 55\% tenían previsto trabajar para poder independizarse. En Del Valle et al. (2003), sólo un 5,8\% de la población entrevistada continuaba estudiando y un 1,9\% trabajaban y estudiaban a la vez. En Inglés (2005) a un 36\% les gustaría seguir estudiando (más las chicas que los chicos); pero la realidad era que el $81,7 \%$ de los sujetos del programa no seguían ningún curso, y de los pocos que estudiaban, una cuarta parte también trabajaba. En FEPA (2007) el 61\% de los cursos iniciados estaban orientados a la formación de un oficio.

De esta transición a la vida adulta nos parece especialmente interesante las ideas expuestas por Melendro (2007) de que a menudo se somete a los alumnos adolescentes a un protagonismo negativo, que si lo aplicamos a los jóvenes tutelados, es más posible que se vuelvan procesos de estigmatización que son percibidos por los jóvenes de forma acusada. Asimismo resalta la importancia de la participación de los jóvenes en su propio itinerario formativo y vital, y la eficacia de los programas de acompañamiento de los jóvenes para su inserción social, identificada también en los estudios de Turró y Cortes (2005), Associació Punt de Referència (2008), ASJTET (2008), Inglés (2005), García Barriocanal et al. (2007). En población general, Gairín et al. (2009) destacan que también son escasas las acciones relacionadas con los procesos de orientación y tutoría dirigidas a los estudiantes de educación secundaria, dificultad percibida así por ellos mismos. Por lo tanto, el acompañamiento y la participación activa de los jóvenes ex-tutelados deberían ser los pilares de los programas de intervención educativa y psicosocial dirigidos a esta población. 


\section{REFLEXIONES FINALES}

En comparación con la población general, los resultados expuestos nos indican que los niños, niñas y adolescentes tutelados, presentan más problemas de rendimiento académico y de conducta, abandonan la escuela prematuramente y obtienen menos graduados de la ESO. A la vez que los jóvenes ex-tutelados llegan a la edad adulta con menos estudios, deben emanciparse mucho antes, ponerse a trabajar y dejar en la mayoría de ocasiones de estudiar. Un cúmulo de factores puede estar influyendo en estos resultados. Quizá se está prestando poca atención desde los servicios de protección social a la infancia en intentar compensar la situación de dificultad escolar con la que muchos entran en el sistema. Quizá los profesionales no acostumbran a tener altas expectativas sobre un buen desempeño de los chicos en la escuela. Quizá la escuela no se ha fijado en ellos como un colectivo susceptible de ser atendido con el diseño de acciones compensatorias. Quizá los departamentos de educación creen que este tema es competencia de los de bienestar, y viceversa. En todo caso, la falta de decisiones políticas concretas orientadas a la recogida sistemática de datos estadísticos no permite conocer con precisión la magnitud e implicaciones del problema y, como consecuencia, no facilita que se debatan y tomen decisiones sobre estrategias de afrontamiento del mismo. Ante esto, los sistemas educativos y de protección social en España deberían, de forma conjunta, empezar a diseñar programas y promover investigaciones para afrontar la falta de igualdad de oportunidades de acceder y mantenerse en el sistema educativo y de formación de la población tutelada, contribuyendo así su bienestar y promoviendo su inclusión social

A pesar de la situación de riesgo de exclusión de la educación y de exclusión social en general de esta población, en la mayoría de los países de la UE no han recibido ninguna atención específica en el ámbito educativo exceptuando el caso del Reino Unido (Casas y Montserrat, 2009), dónde se han realizado cambios legislativos y políticos en esta dirección, partiendo de la disponibilidad de datos sistemáticos, lo que está permitiendo el desarrollo de programas de intervención concretos. 


\section{NOTAS}

1. Esta revisión forma parte de una investigación dentro del $7^{\circ}$ Programa Marco de la Unión Europea, centrada en los itinerarios educativos de los jóvenes que han estado tutelados una parte o toda su infancia por los servicios de protección (http://tcru.ioe.ac.uk/yippee). 


\section{REFERENCIAS BIBLIOGRÁFICAS}

Àrea de Suport als Joves Tutelats i Ex-tutelats (ASJTET) (2008). Estudi sobre els joves residents en recursos de la DGAIA. L'ASJTET, principal recurs de sortida. Generalitat de Catalunya. Butlletí d'Infància, 21,2-7.

Associació Punt de Referència (2008). Acompanyament voluntari $i$ vincle afectiu. Experiències d'acompanyament amb joves ex-tutelats. Barcelona: Associació Punt de Referencia.

Bravo, A. y Del Valle, J.F. (2001). Evaluación de la integración social en acogimiento residencial. Psicothema, 13(2), 197-204.

Bravo, A. y Del Valle, J.F. (2003). Las redes de apoyo social de los adolescentes acogidos en residencias de protección. Un análisis comparativo con población normativa. Psicothema, 15(1), 136-142.

Casas, F. (1998). Infancia: Perspectivas psicosociales. Barcelona: Paidós.

Casas, F. y Montserrat, C. (2009). Sistema educativo e igualdad de oportunidades entre los jóvenes tutelados: estudios recientes en el Reino Unido. Psicothema.21(4), 543-547.

Commission of the European Communities (2008). Progress towards the Lisbon objectives in education and training. Disponible en: http://ec.europa. eu/education/policies/2010/doc/progres s08/report_en.pdf

Council of the EU (2006). National report on strategies for social protection and social inclusion 2006-2008. Disponible en: http://ec.europa.eu/employment_ social/spsi/docs/social_inclusion/ 2006/nap/malta_en.pdf

Consell Superior d'Avaluació del Sistema Educatiu (CSASE) (2007a). Quaderns d'avaluació, 9: Estudi PISA 2006. Avançament de resultats. Barcelona: Generalitat de Catalunya. Departament d'Educació.

Consell Superior d'Avaluació del Sistema Educatiu (CSASE) (2007b). Informes d'Avaluació 10: Informe per a la millora dels resultats del sistema educatiu a Catalunya. Barcelona: Generalitat de Catalunya. Departament d'Educació.

Del Valle J., López, M., Montserrat, C. y Bravo, A. (2007). Evaluación de resultados de la medida de acogimiento familiar en España. Madrid: Ministerio de Trabajo y Asuntos Sociales.

Del Valle, J.F., Álvarez-Baz, E. y Bravo, A. (2003). Evaluación de resultados a largo plazo en acogimiento residencial de protección a la infancia. Infancia $y$ Aprendizaje 26(2), 235-249.

European Commission (2007). Eurybase. The Information Database on Education Systems in Europe. The Education System in Spain. 2006/07. Disponible en: http://www.eurydice.org/ressources/ eurydice/eurybase/pdf/0_integral/ES_ EN.pdf

Federació d'Entitats amb Projectes i Pisos Assistits (FEPA) (2007). Memòria 2006. Barcelona: FEPA i Diputació de Barcelona.

Gairín, J., Muñoz, JL., Feixas, M. y Guillamón, C. (2009). La transición Secundaria-Universidad y la incorporación a la Universidad. La acogida de los estudiantes. Revista Española de Pedagogía, 242.

García Barriocanal, C., Imaña, A. y de la Herrán, A. (2007). El acogimiento residencial como medida de protección al menor. Madrid: Defensor del Menor en la Comunidad de Madrid. 
Inglés, A. (dir.) (2005). Aprendiendo a volar. Estudio para el análisis de los programas europeos Mentor 15 y Ulises dedicados al proceso de socialización de adolescentes y jóvenes tutelados que han alcanzado la mayoría de edad o están cerca de ella. Murcia: Fundación Diagrama.

Lamarca, I. y Barceló, F. (2006). Personas menores en situaciones de especial vulnerabilidad: retos para una intervención eficaz. Revista de Psicodidáctica, $11,1,25-36$.

Martín, E., Muñoz, C., Rodríguez, T. y Pérez, Y. (2008). De la residencia a la escuela: la integración social de los menores en acogimiento residencial con el grupo de iguales en el contexto escolar. Psicothema, 20(3), 376-382.

Melendro, M. (dir.) (2007). Estrategias educativas con adolescentes y jóvenes en dificultad social. Madrid: UNED.

Melendro, M (2004). Educación y globalización: educar para la conciencia de los límites, Educación XX1 (6), 235-246.

Merino, R. y García, M. (2007). Itineraris de formació $i$ inserció laboral dels joves a Catalunya. Barcelona: Fundació Jaume Bofill.

Montserrat, C. y Casas, F. (2006). Kinship foster care from the perspective of quality of life: Research on the satisfaction of the stakeholders. Applied Research in quality of life, 1, 227-237.

Navarrete, L. (dir.) (2007). Jóvenes y fracaso escolar en España. Madrid: Instituto de la Juventud (INJUVE).

Panchón (dir.) (1999). Situación de menores de 16 a 18 años en centros de protección. Disponible en: http://www. dulac.org/PDFs/resinvmenores.pdf

Rodríguez Gómez, R. (2006). La integración social y laboral de jóvenes que han estado tutelados y tuteladas por la Junta de Andalucía y han cumplido la mayoría de edad. Revista de Estudios de Juventud, 74, 251-277.

Secretaria de Joventut (2008). Vulnerabilitat dels joves davant la crisi. Bacelona: Generalitat de Catalunya, Secretaria de Joventut.

Turro, C. y Cortes, J. (2004). La relación de ayuda con jóvenes ex-tutelados por la administración catalana: una intervención psicosocial basada en la formación y apoyo de voluntarios que favorezcan su integración social. Psykhe, 13,2, 159-171.

Uriarte, J.D. (2006). Construir la resiliencia en la escuela. Revista de psicodidáctica 11,(1), 7-24. 


\section{PALABRAS CLAVE}

Niños y adolescentes tutelados, jóvenes ex-tutelados, sistema educativo, sistema de protección a la infancia, exclusión social, igualdad de oportunidades.

\section{KEY WORDS}

Children in care, young people leaving care, educational system, child protection system, social exclusion, equal opportunities.

\section{PERFIL ACADÉMICO DE LOS AUTORES}

Dra. Carme Montserrat Boada. Pedagoga por la Universidad de Barcelona, se doctoró en Psicología por la Universidad de Girona, donde trabaja de profesora. Ha realizado varios estudios y publicaciones tanto en el ámbito educativo como de protección social y actualmente participa en una investigación en cinco países europeos, dentro del $7^{\circ}$ Programa Marco de la Unión Europea, sobre los factores que pueden ayudar a los jóvenes tutelados a seguir sus estudios después de la escolaridad obligatoria. Colabora con el Consejo de Europa como experta en prevención de la violencia infantil.

Dr. Ferran Casas Aznar. Facultad de Educación y Psicología. Universidad de Girona. Catedrático de psicología social en la Universidad de Girona y coordinador del ERIDIQV (Equipo de Investigaciones sobre la Infancia, sus Derechos y su Calidad de Vida), adscrito al Instituto de Investigaciones sobre Calidad de Vida de la misma Universidad, del cual ha sido director más de 10 años. Durante cuatro años fue Presidente del Comité de Expertos Coordinador del Proyecto Políticas de Infancia del Consejo de Europa; durante tres, director del Centro de Estudios del Menor del Ministerio de Asuntos Sociales; y durante otros tres, el Director del Programa de Atención a la Infancia en Alto Riesgo Social de la Generalitat de Catalunya; así como profesor de psicología social de la Universidad de Barcelona.

Dirección de los autores: Universidad de Girona.

Facultad de Educación y Psicología.

C/Creu, 2 - 17071 Girona

E-mail: carme.montserrat@udg.edu

ferran.casas@udg.edu

Fecha de Recepción del artículo: 1. Agosto. 2009

Fecha de Revisión del artículo: 2. Noviembre. 2009

Fecha de aceptación del artículo: 10. Noviembre. 2009 